
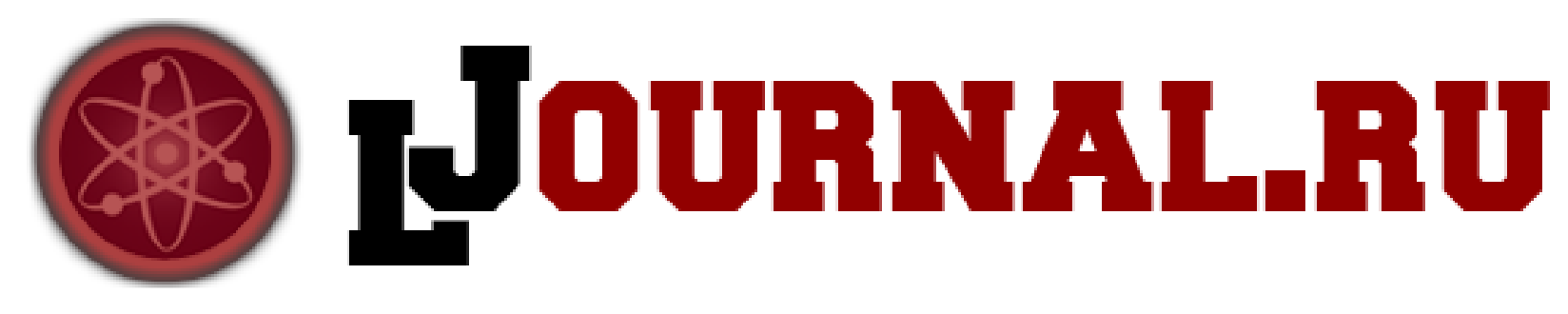

Муравьев К.А.

Филиал ТюмГНГУ в г. Сургуте

Сургут, Россия

doi: 10.18411/1j2016-2-14

\title{
Оценка эксплуатационной работоспособности нефтепромысловых металлоконструкций
}

В статье приведень сведения о новой методике инженерной оценки эксплуатационной надежности нефтепромысловых транспортных систем

Актуальность темы. Топливно-энергетический комплекс Украины является основой развития экономики и оказывает существенное влияние на рост научно-технического прогресса, интенсификацию производственных процессов, повышение и совершенствование их технологического уровня, а также во многом определяет темпы роста национального дохода страны. Несмотря на снижение объемов добычи нефти, вызванное экономическими трудностями переходного периода, в нашей стране и колебанием цен на мировом рынке, основная часть транспортных систем по перекачке и хранению нефти и нефтепродуктов продолжает активно эксплуатироваться.

Анализ состояния основного фонда нефтедобывающего и транспортного комплекса страны показывает, что одной из основных проблем эксплуатации является его физический и моральный износ. Решение этой важной практической проблемы сопряжено с огромными капиталовложениями и, по нашему мнению, в ближайшие годы невыполнимо. В связи с этим возрастает 
роль капитального ремонта с заменой отдельных участков трубопроводных конструкций, насосных станций, задвижек, стальных вертикальных резервуаров для хранения нефти на трубы и корпусные элементы с повышенными коррозионно-механическими характеристиками.

Проблема обеспечения требуемой эксплуатационной надежности нефтепромыслового оборудования и инженерных металлоконструкций становится с каждым годом все более актуальной в связи с опережающей тенденцией старения оборудования по сравнению с темпами технического перевооружения нефтяной отрасли Украины. Поэтому одной из первоочередных задач в сложившихся сложных условиях, когда обновление физически и морально устаревших основных фондов из-за финансовых затруднений происходит в ограниченных объемах, является сохранение и продление срока службы нефтепромысловых систем путем применения эффективных методов и способов увеличения межремонтных циклов.

Указанная проблема усугубляется отсутствием научно-обоснованных концепций технической диагностики и методов определения рабочего ресурса и недостаточной эффективностью традиционных методов и средств неразрушающего контроля. В связи с этим в настоящее время важное значение приобретают расчетные методы с использование критериев оценки надежности элементов сложных конструкций на базе теории вероятности [5,6]. Поэтому целью данной работы являлась разработка методики инженерной оценки эксплуатационной надежности нефтепромысловых трубопроводных конструкций и оборудования, которая основывалась бы на результатах эксплуатационных исследований (наблюдений), необходимых и достаточных для определения надежности и остаточного рабочего ресурса отдельных элементов конструкции и в целом нефтетранспортной системы. При этом под элементом, например трубопроводной конструкции, следует понимать как отдельные узлы или агрегаты, так и любую ее систему, подсистему, деталь, компонент и т.д. 


\section{Результаты исследований и их обсуждение}

\section{1. Показатели надежности, которые определяются}

Известно [5], что надежность - это свойство объекта сохранять во времени в установленных пределах значения всех параметров, характеризующих способность выполнять требуемые функции в заданных режимах и условиях применения техническогго обслуживания, ремонтов, хранения и транспортировки. В данном определении имеются следующие особенности.

Во-первых, подчеркнута непрерывность выполнения объектом заданных функций. В этом аспекте нет смысла говорить о надежности объекта, например, во время проведения на нем планово-предупредительных работ, ремонтов, замены старого оборудования, освидетельствований и других мероприятий ибо в это время объект не выполняет своих функций.

Во-вторых, в определение надежности включено понятие установленные пределы, т.е. сложная система при отказе отдельных элементов или подсистем сохраняет свою работоспособность.

В-третьих, надежность объекта целесообразно вычислять за определенные промежутки времени.

Надежность как сложное свойство применительно к рассматриваемой в данной работе транспортной системы и ее элементов и условий их эксплуатации состоит из сочетаний свойств безотказности, ремонтопригодности, долговечности и сохранности. Учитывая специфику условий эксплуатации трубопроводных коммуникаций по транспорту нефти и емкостей для еe хранения, организацию технического обслуживания и ремонта в полевых (трассовых) и базовых (цеховых) условиях, нами рекомендуется использовать в расчетах следующие показатели надежности:

a) группа показателей безотказности, характеризующие свойства объекта исполнять требуемые функции в определенных условиях в течение заданного интервала времени и наработке, в частности: 
- вероятность безотказной работы $\mathrm{F}(\mathrm{t})$ - вероятность того, что в пределах заданной наработки отказ объекта не возникает. Функция $\mathrm{F}(\mathrm{t})$ является основной количественной характеристикой безотказности объекта на заданном временном интервале;

- интенсивность отказов $\lambda(\mathrm{t})$ - условная плотность вероятности возникновения отказа объекта (элемента), которая определяется при условии, что до этого момента отказа не возникало;

$\lambda(\mathrm{t})=\Delta \mathrm{n}(\Delta \mathrm{t}) / \mathrm{N}(\mathrm{t}) \Delta \mathrm{t}$,

где $\Delta \mathrm{n}(\Delta \mathrm{t})$ - число отказов объекта за промежуток времени от $(\mathrm{t}-\Delta \mathrm{t} / 2)$ до $\mathrm{t}+$ $\Delta \mathrm{t} / 2) ; \mathrm{N}(\mathrm{t})=\left(\mathrm{N}_{\mathrm{i}-1}+\mathrm{N}_{\mathrm{i}}\right) / 2$,

здесь $\mathrm{N}_{\mathrm{i}-1}$ - число исправно работающих объектов в начале интервала времени $\Delta \mathrm{t}$

, $\mathrm{N}_{\mathrm{i}}$ - число исправно работающих объектов в конце интервала времени $\Delta \mathrm{t}$.

- средняя наработка на отказ Тср - это отношение суммарной наработки объекта к математическому ожиданию числа его отказов в течение этой наработки

$\mathrm{Tcp}=\frac{1}{n} \cdot \sum_{i=1}^{n} \mathrm{tcpi}$,

где $\mathrm{t}_{\text {срі }}$ - время исправной работы между $(\mathrm{i}-1)$-м и $\mathrm{i}$-м отказами объекта, n - число отказов объекта ;

- параметр потока отказов w -это отношение математически ожидаемого (среднего) числа отказов восстанавливаемого объекта за произвольно малую его наработку к значению этой наработки. Статистический параметр потока отказов можно определять по формуле:

$\mathrm{qw}(\mathrm{t})=\Delta \mathrm{n}_{\mathrm{i}}(\Delta \mathrm{t}) / \mathrm{N}_{\mathrm{i}} \Delta \mathrm{t}$, где $\Delta \mathrm{n}_{\mathrm{i}}(\Delta \mathrm{t})$ - общее число отказов восстанавливаемого объекта за интервал времени $(\mathrm{t}-\Delta \mathrm{t} / 2)$ до $(\mathrm{t}+\Delta \mathrm{t} / 2)$.

б) группа показателей долговечности, которые характеризуют свойтства объекта исполнять требуемые функции до перехода в предельное состояние при установленной системе технического обслуживания и ремонта, в частности:

- средний ресурс Тр -это математическое ожидание ресурса; 
- гамма-процентный ресурс Т $\gamma$ - это наработка, в течение которой объект не достигает предельного состояния с заданной вероятностью $\gamma$, выраженной в процентах.

Гамма-процентный ресурс определяется по формуле:

$$
\mathrm{T}_{\gamma}=1-\int_{0}^{t} f(\mathrm{t}) \mathrm{dt}=\gamma / 100 \text {. }
$$

Ремонтопригодность трубопроводных конструкций и их отдельных элементов (узлов) и инженерного оборудования и емкостей для подготовки и хранения нефти - как свойство объекта, заключающееся в приспособленности к предупреждению и обнаружению причин отказов (повреждений) и восстановлению работоспособного состояния путем проведения технического обслуживания и ремонтов, следует рассматривать при внеплановых (срочных) ремонтах. Поскольку остановка и подготовка нефтяных транспортных коммуникаций, хранилищ, насосных станций и другого оборудования осуществляется, как правило, в плановом порядке независимо от технического состояния отдельных узлов (агрегатов), то показатели ремонтопригодности (вероятность восстановления, средняя продолжительность восстановления и др.) в данной работе не будут рассматриваться.

2. Методика сбора и обработки информации о техническом состоянии нефтетранспортных систем и инженерного оборудования

Выбор показателей надежности транспортных коммуникационных систем ставит задачу установления критериев отказа и предельного состояния элементов (узлов, агрегатов) коммуникационных (транспортных) систем с учетом конструктивного решения характера его эксплуатации и последствий отказа. Предельное состояние элемента определяется невозможностью его дальнейшей эксплуатации или недопустимым снижением эффективности, нарушением требований безопасности, угрозой жизни обслуживающего персонала. животному миру и растительной среде.

Методика сбора и первоначальной обработки информации о техническом 
состоянии объектов нефтетранспортной системы включает:

- подготовку к сбору информации о техническом состоянии;

- обследование технического состояния оттельных объектов нефтетранспортной системы или непосредственно элементов, надежность которых рассматривается;

- первоначальную обработку полученной информации.

Подготовка к сбору информации о техническом состоянии трубопроводной конструкции и инженерного вспомогательного оборудования проводится с целью выявления потенциально возможных отказов путем анализа нормативно-технической документации, в частности, строительных норм и правил (СНиП), а также ведомственных нормативных документов (BCH), регламентирующих строительство и эксплуатацию ответственных конструкций. В результате анализа СНиП и ВСН разрабатывается схема технического осмотра (обследования) и фиксации отказов.

Обследование технического состояния проводится с целью выявления непосредственно отказов или характерных повреждений, которые определяют предельное состояние элемента трубопроводной системы. Трубопроводы, несмотря на конструктивную внешнюю простоту, существенно отличаются от других сооружений сложной схемой взаимодействия силовых факторов, разновидностью нагружения, неопределенностью напряженнодеформированного состояния (НДС), масштабностью и т.п. Срок службы, который заложен в проектах, составляет от 12 до 30 лет [7]. Так, в НГДУ “Надвирнанафтогаз” ВАТ “Укрнафта" примерно $18 \%$ нефтепроводов эксплуатируется почти 30 лет, 22\% - свыше 20 лет, т.е. фактически исчерпан их pecypc [8].

Информация, собранная в процессе проведения обследования оборудования и отмеченная в картах обследования, проходит первичную качественную и в дальнейшем количественную обработку, а также анализируется с целью выявления причин появления неисправностей, дефектов 
и Т.П.

Целью первичной обработки является отсев явно недостоверного материала, оценка полноты и однородности информации, которая осталась, ее классификация и ранжирование,

Ранжирование материалов обследования состоит в систематизации первичной информации в порядке возрастания срока эксплуатации на момент проведения обследования. При этом учитываются только основные виды отказов и соответствующие им модели:

- конструктивные (усталость, закономерный износ, влияние неучтенных расчетных факторов);

- технологические (присутствие скрытых дефектов, низкие характеристики качества изготовления);

- эксплуатационные (нарушение правил эксплуатации, обслуживания и ремонта).

При возникновении в одной конструктивной зоне элемента разных по своей физической природе повреждений, последние учитываются в качестве отдельных.

Из литературы [9-11] и практики известно, что надежность и ресурс оборудования определяются, в основном, зонами концентрации напряжений, в которых уровень фактических наряжений может достигать предела текучести и выше.

Тенденция перехода от традиционной дефектоскопии к технической диагностике с применением комплексного подхода, включающего определение параметров дефектов, оценку распределения внутренних (остаточных) напряжений, определение фактических структурно-механических характеристик металла, сдерживается, в первую очередь, низкой эффективностью существующих методов и средств контроля напряженно-деформированного состояния оборудования.

На основе опыта эксплуатации трубопроводных транспортных систем и 
других инженерных коммуникаций и наблюдений за появлениями отказов (повреждений) принимается, что вероятность появления отказов (повреждений) в относительно малом интервале времени $\Delta \mathrm{t}$ пропорциональна длине этого интервала. При этом коэффициент пропорциональности равен $\lambda$. Это дает возможность длительность срока службы элемента определить до появления отказа из непараметрического выражения вероятности безотказной работы [2]:

$$
\mathrm{P}(\mathrm{t})=1-\mathrm{F}(\mathrm{t})-\exp \left(-\int_{0}^{t} \lambda(\tau) \mathrm{d} \tau\right),
$$

где $\lambda(\mathrm{t})$ - в каждый момент времени является суммой интенсивностей отказов iтых элементов:

$$
\int_{0}^{t} \lambda(\tau) \mathrm{d} \tau=\sum \int_{0}^{t} \lambda_{\mathrm{i}}(\tau) \mathrm{d} \tau
$$

Количественный анализ информации содержит характерную оценку неисправности отдельных элементов или узлов (агрегатов), а также расчет показателей поврежденности, соответствующих формулам (1) и (2).

Первичная обработка статистических данных может быть представлена вычислительным процессом, исходными данными для которого являются срок службы ( ti ), количество обследованных (Ni) и поврежденных (ni) элементов в i-том интервале, который состоит в определении:

- величины частоты появления отказов (неисправностей) в і-том интервале

$$
\mathrm{q}_{\mathrm{i}}=\mathrm{n}_{\mathrm{i}} / \mathrm{N}_{\mathrm{i}}
$$

- собранной интервальной частоты отказов (неисправностей) для і-того интервала

$$
\mathrm{r}_{\mathrm{i}}=\sum_{k+1}^{i} \mathrm{q}_{\mathrm{k}}=\sum_{k+1}^{i} \mathrm{n}_{\mathrm{k}} / \mathrm{N}_{\mathrm{k}}
$$


- - эмпирической вероятности отказа элемента (узла агрегата) за і-тый срок службы

$$
\mathrm{Q}_{\mathrm{i}}=1-\exp \left(-\mathrm{r}_{\mathrm{i}}\right)=1-\exp \left(-\sum_{k+1}^{i} \mathrm{n}_{\mathrm{k}} / \mathrm{N}_{\mathrm{k}}\right) .
$$

Функция $\mathrm{Q}(\mathrm{t})$ представляет собой интегральную функцию распределения случайной величины, т.е. $\mathrm{Q}(\mathrm{t})=\mathrm{P}(\mathrm{t})$. Если функция $\mathrm{Q}(\mathrm{t})$ дифференцируема, то производная от интегральной функции распределения есть дифференциальный закон (плотность) распределения случайной величины Т- времени исправной работы, т.е. статистическая вероятность отказа равна:

$$
\mathrm{Q}(\mathrm{t})=\lim \frac{\sum_{i=1}^{t / \Delta t} n_{i}}{N_{0}} \text { при } \Delta \mathrm{t} \rightarrow 0 \text { и } \mathrm{N}_{0} \rightarrow \infty,
$$

где N0 - число объектов в начале испытаний; ni - число отказавших объектов в интервале времени $\Delta \mathrm{t} ; \mathrm{t}-$ время, для которого определяется вероятность безотказной работы конструкции.

Определение необходимого минимального количества эксплуатационных наблюдений в заданном временном интервале $\Delta \mathrm{t}$ осуществляется табличным методом [3] и связано с выбором значений достоверной вероятности, величиной относительной ошибки и с законом распределения исследуемого показателя. Для получения достоверных оценок объем выборки должен быть достаточно большим.

Обычно размер выборки, необходимый для проведения экспериментов, определяется выражением:

$$
\mathrm{N}_{1} \geq\left(\mathrm{t}_{\alpha}^{2} \sigma^{2} / \Delta^{2}\right) \cdot \mathrm{C}_{\mathrm{k}+\mathrm{d}}^{\mathrm{k}},
$$

где $\mathrm{t} 2 \alpha \sigma 2 / \Delta 2=\mathrm{n}-$ количество объектов для одного эксперимента; t $\alpha$ - коэффициент, характеризующий вероятность того, что расхождение не будет больше $\Delta$ (при $\mathrm{t} \alpha=2$ вероятность $\alpha=0.95$; при $\mathrm{t} \alpha=3$ вероятность $\alpha=$ 0.997); $\sigma-$ среднее квадратичное отклонение параметра в генеральной совокупности; $\Delta-$ минимальное расхождение между выборочными и 
генеральными средними арифметическими, соответствующими заданной вероятности $\alpha ; \quad \mathrm{Ckk}+\mathrm{d}-$ коэффициент, зависящий от числа исследуемых факторов (k) и порядка уравнения регрессии (d), выбирается по таблицам [3].

\section{3. Алгоритм определения показателей надежности и вероятности} безотказной работы трубопроводных конструкций и инженерного нефтепромыслового оборудования

Для оценки адекватности выборковой совокупности генеральной используют метод подбора теоретического закона распределения вероятности работы конструкции (элемента) в исправном состоянии. Учитывая, что основные повреждения в стальных конструкциях (как показывапет практика), приводящие к отказам и авариям, носят усталостный характер, то в качестве теоретического закона принимается рапределение Вейбулла [4], отдельными случаями которого является нормальный и экспоненциальный законы:

$$
\mathrm{F}(\mathrm{t})=1-\exp \left(-\left(\mathrm{t} / \mathrm{t}_{0}\right)^{\mathrm{m}}\right),
$$

где $\mathrm{t}$ - время наработки;

$\mathrm{m}$ - параметр формы распределения;

t0 - параметр масштаба распределения.

Параметры m и t0 определяются по таблицам [4].

В качестве метода статистического оценивания параметров распределения используется метод максимальной правдоподобности. Для этого формула распределения Вейбулла после перестановки членов и двойного логарифмирования приобретает вид:

$$
\ln (\mathrm{t})=\frac{1}{m} \ln \left(\ln \left(\frac{1}{1-F(t)}\right)\right)+\ln \left(\mathrm{t}_{0}\right)
$$

или

$$
\mathrm{y}=\mathrm{a}+\mathrm{bz}
$$

где

$$
\mathrm{y}=\ln (\mathrm{t}) ; \quad \mathrm{z}=\ln \left(\ln \left(\frac{1}{1-F(t)}\right)\right)
$$




$$
\mathrm{b}=1 / \mathrm{m} ; \quad \mathrm{a}=\ln \left(\mathrm{t}_{0}\right) .
$$

Выравнивание экспериментальных данных за формулой (8) производится методом наименьших квадратов, при этом вместо $1-\mathrm{F}(\mathrm{t})$ подставляют эмпирические значения Qi , которые определяются по формуле (5):

$$
\mathrm{U}=\sum_{i=1}^{n}\left(\mathrm{y}_{\mathrm{i}}-(\mathrm{a}+\mathrm{b} \mathrm{z})\right)^{2}
$$

где $\mathrm{n}$ - число интервалов, причем само решение задачи сводится к определению таких значений коэффициентов а и b, которые минимизируют сумму отклонений эмпирических значений и значений, определенных по формуле (9).

Для этого необходимо найти отдельные производные функции (9) за коэффициентами а и b и приравнять их нулю:

$$
\begin{array}{r}
\mathrm{dU} / \mathrm{da}=\sum_{i=1}^{n}\left(\mathrm{y}_{\mathrm{i}}-(\mathrm{a}+\mathrm{b} \mathrm{z})\right)=0 ; \\
\mathrm{dU} / \mathrm{db}=\sum_{i=1}^{n}\left(\mathrm{y}_{\mathrm{i}}-(\mathrm{a}+\mathrm{b} \mathrm{z})\right) \mathrm{z}_{\mathrm{i}}=0 .
\end{array}
$$

Преобразив полученную систему уравнений, получим:

$$
\begin{array}{r}
\mathrm{a} \cdot \mathrm{n}+\sum_{i=1}^{n} \mathrm{z}_{\mathrm{i}}=\sum_{i=1}^{n} \mathrm{y}_{\mathrm{i}} ; \\
\mathrm{a} \sum_{i=1}^{n} \mathrm{z}_{\mathrm{i}}+\mathrm{b} \sum_{i=1}^{n} \mathrm{z}_{\mathrm{i}}^{2}=\sum_{i=1}^{n} \mathrm{y}_{\mathrm{i}} \mathrm{z}_{\mathrm{i}}
\end{array}
$$

решая которую найдем параметры а и b:

$$
\begin{aligned}
\mathrm{a} & =\left(\sum_{i=1}^{n} \mathrm{y}_{\mathrm{i}} \cdot \sum_{i=1}^{n} \mathrm{z}_{\mathrm{i}}^{2}-\sum_{i=1}^{n} \mathrm{z}_{\mathrm{i}} \mathrm{y}_{\mathrm{i}} \cdot \sum_{i=1}^{n} \mathrm{z}_{\mathrm{i}}\right) /\left(\mathrm{n} \sum_{i=1}^{n} \mathrm{z}_{\mathrm{i}}-\left(\sum_{i=1}^{n} \mathrm{z}_{\mathrm{i}}\right)^{2}\right) \\
\mathrm{b} & =\left(\mathrm{n} \sum_{i=1}^{n} \mathrm{z}_{\mathrm{i}} \mathrm{y}_{\mathrm{i}}-\sum_{i=1}^{n} \mathrm{z}_{\mathrm{i}} \cdot \sum_{i=1}^{n} \mathrm{y}_{\mathrm{i}}\right) /\left(\mathrm{n} \sum_{i=1}^{n} \mathrm{z}_{\mathrm{i}}-\left(\sum_{i=1}^{n} \mathrm{z}_{\mathrm{i}}\right)^{2}\right) .
\end{aligned}
$$

Тогда параметры распределения Вейбулла определяют из выражений $\mathrm{m}=1 / \mathrm{b} ; \mathrm{t}_{0}$ $=\exp (\mathrm{a})$

Для сравнения эмпирических и теоретических функций распределения 
используется критерий схождения Пирсона, являющийся наиболее обоснованным при большом количестве наблюдений. Его обоснованность состоит в том, что он почти всегда опровергает неверную гипотезу и забеспечивает минимальную ошибку в принятии неверной гипотезы.

Количественные значения показателей надежности трубопроводных конструкций, а также отдельных элементов (узлов, агрегатов) определяются по формуле [4] :

- вероятность работы конструкции в исправном состоянии

$$
\mathrm{F}(\mathrm{t})=\exp \left(-\left(\mathrm{t} / \mathrm{t}_{0}\right)^{\mathrm{m}}\right) \text {; }
$$

$$
\begin{aligned}
& \lambda(\mathrm{t})=\left(\mathrm{m} / \mathrm{t}_{0}\right) \cdot\left(\mathrm{t} / \mathrm{t}_{0}\right)^{\mathrm{m}-1} ; \\
& \text { отказов } \\
& \text { - - } \\
& \text { гамма-процентный } \\
& \text { (повреждений) } \\
& \mathrm{T}_{\mathrm{j}}=\mathrm{m} \sqrt{ } \ln \frac{\gamma}{100} \\
& \mathrm{w}(\Delta \mathrm{t})=\frac{1}{m}\left(\exp \left(-\left(\mathrm{t}_{\mathrm{i}} / \mathrm{m}\right)^{\mathrm{m}}\right)-\exp \left(-\left(\mathrm{t}_{\mathrm{i}-1} / \mathrm{t}_{0}\right)^{\mathrm{m}}\right)\right) ; \\
& \text { - с средняя наработка на отказ (повреждения) } \\
& \mathrm{T}_{\mathrm{cp}}=\mathrm{t}_{0} \cdot \Gamma(1+1 / \mathrm{m}),
\end{aligned}
$$

где $\Gamma(1+1 / \mathrm{m})$ - гамма-функция, которая определяется по таблицам специальных функций [3].

Для оценки долговечности трубопроводных транспортных конструкций необходимо определить их ресурс и срок службы, которые являются наиболее важными показателями надежности. Прогнозирование остаточного ресурса на стадии эксплуатации связано с оперативной технической диагностикой, а также постоянным мониторингом состояния конструкции в рабочих (нагруженных ) режимах. 


\section{4. Оценка остаточного (рабочего) ресурса промысловых}

\section{нефтепроводов}

Известно [5], что безопасность определяется механическими напряжениями в стенке трубы. При эксплуатации трубопровод подвергается следеющим факторам воздействий:

- внутреннее давление транспортируемого продукта,

- упругий изгиб (искривление трубопровода) в вертикальной и горизонтальной плоскостях,

- температурное воздейтсвие.

Внутреннее давление в трубе вызывает в стенке трубы кольцевые и продольные напряжения, которые определяются следующими формулами: кольцевое напряжение

$$
\sigma^{\mathrm{p}}{ }_{\text {кц }}=\left[\mathrm{nP}\left(\mathrm{D}-2 \delta_{\mathrm{T}}\right)\right] / 2 \delta_{\mathrm{T}},
$$

где $\mathrm{P}$ - внутреннее давление в трубопроводе в процессе ремонта, Па; $\delta_{\mathrm{T}}-$ толщина стенки трубы (фактическая), м; D - наружный диаметр трубы, м; n коэффициент надежности по нагрузке - внутреннему рабочему давлению в трубопроводе;

\section{продольное напряжение}

$$
\sigma_{\text {пр }}^{\mathrm{p}}=\mu \sigma_{\text {кц }}^{\mathrm{p}}-\alpha \mathrm{E}\left(\mathrm{T}_{\mathrm{c}}-\mathrm{T}_{\ni}\right)=\left[\mu \mathrm{nP}\left(\mathrm{D}-2 \delta_{\mathrm{T}}\right) / 2 \delta_{\mathrm{T}}\right]-\alpha \mathrm{E}\left(\mathrm{T}_{\mathrm{c}}-\mathrm{T}_{\epsilon}\right),
$$

где $\alpha=12 \cdot 10^{-6} 1 /$ град - коэффициент температурного расширения стали; $\mathrm{E}=206$ $\cdot 10^{9}$ Па - модуль продольной упругости стали; $\mathrm{T}_{\mathrm{c}}$ - температура грунта при укладке (при строительстве); $\mathrm{T}_{\ni}$ - температура грунта при ремонтных работах, $\mu$ $=0.3-$ коэффициент поперечной деформации.

В процессе нормальной эксплуатации трубопровода (а также в процессе ремонтных работ) суммарные напряжения не должны превышать допустимых напряжений $\sigma_{\text {доп }}=[\sigma]$, определенных с учетом параметров фактического технического состояния трубопровода, включая механические свойства стали срок эксплуатации, уровень дефектности, категорию безопасности, коэффициенты надежности. 
Допустимые напряжения стенки трубы определяются из требований, изложенных в СНиП 2.05.06-85.

В расчетах допустимых напряжений используются расчетные сопротивления металла растяжению (сжатию) $\mathrm{R}_{1}$ и $\mathrm{R}_{2}$ :

$\mathrm{R}_{1}=\left(\sigma_{\mathrm{B}} \mathrm{m}\right) /\left(\mathrm{k}_{1} \mathrm{k}_{\mathrm{H}}\right)$

$\mathrm{R}_{2}=\left(\sigma_{\mathrm{n}} \mathrm{m}\right) /\left(\mathrm{k}_{2} \mathrm{k}_{\mathrm{H}}\right)$

где $\sigma_{\mathrm{B}}-$ минимальное значение предела прочности; $\sigma_{\mathrm{T}}-$ минимальное значение предела текучести; $\mathrm{m}$ - коэффициент условий работы ; $\mathrm{k}_{1}$ и $\mathrm{k}_{2}$ - коэффициенты надежности по материалу; kн - коэффициент надежности по назначению трубопровода.

Значения $\sigma_{\mathrm{T}}$ и $\sigma_{\mathrm{B}}$ для новых труб определяются из сертификатов для длительно эксплуатируемых трубопроводов - экспериментально или расчетом с учетом эффекта старения металла труб и сварных швов.

Для выполнения оценочных расчетов принимаем такие значения перечисленных коэффициентов, которые соответствуют наибольшему запасу прочности.

Значение коэффициента $\mathrm{m}$ зависит от категории участка и определяется по таблице 1 СНиП 2.05.06-85. Наименьшее значение $\mathrm{m}=0.75$ для обследуемых трубопроводов категории 1 и 11.

Коэффициент $\mathrm{k}_{1}$ зависит от особенностей технологии изготовления труб и определяется по таблице 9 СНиП 2.05.06-85. Наибольшее значение $\mathrm{k}_{1}=1.55$.

Коэффициент $\mathrm{k}_{2}$ зависит от особенностей технологии изготовления труб и отношения $\sigma_{\mathrm{T}} / \sigma_{\mathrm{B}}$, определяется по таблице 10 СНиП 2.05.06-85. Наибольшее значение $\mathrm{k}_{2}=1.15$.

Коэффициент $\mathrm{k}_{\mathrm{H}}$ зависит от диаметра трубопровода, определяется по таблице 11 СНиП 2.05.06-85. Для нефтепроводов с давлением до $5.5 \mathrm{MПа}$ наибольшее значение $\mathrm{k}_{\mathrm{H}}=1.00$.

Допустимые напряжения определяются следующими условиями.

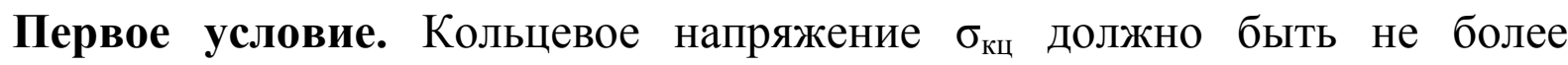


допустимого значения $\left[\sigma_{\text {кц }}\right]_{1}$ :

$\sigma_{\text {кц }} \leq\left[\sigma_{\text {кц }}\right]_{1}=\mathrm{R}_{1} \psi_{1} / \mathrm{n}_{\mathrm{p}}$.

Здесь $\mathrm{n}_{\mathrm{p}}$ - коэффициент надежности по внутреннему давлению в трубопроводе, наибольшее значение $\mathrm{n}_{\mathrm{p}}=1.15 ; \psi_{1}$ - коэффициент, учитывающий двухосное напряженное состояние стенки трубопровода, определяемый по формуле:

$$
\psi_{1}=\sqrt{1-0.75^{2}-0.5 \xi},
$$

где $\xi$ - коэффициент двухосности наряженного состояния, определяемый следующим образом: $\xi=0$ для растягивающих продольных напряжений, $\xi=\mathrm{R}_{1}$. $\left[\sigma_{\text {пр }}\right]_{1}-$ для сжимающих продольных напряжений.

Второе условие. Продольное напряжение $\sigma_{\text {пр }}$ по абсолютной величине не должно превышать допустимого значения $\left[\sigma_{\text {пр }}\right]_{2}$, определяемого по формуле:

$\sigma_{\text {пр }} \leq\left[\sigma_{\text {пр } 2}\right]=\mathrm{R}_{1} \cdot \psi_{2}$.

Здесь $\psi_{2}-$ коэффициент, учитывающий двухосное напряженное состояние стенки трубопровода, определяемый по формуле

$$
\Psi_{2}=\sqrt{ } 1-0.75 \eta^{2}-0.5 \eta,
$$

где $\eta$ - коэффициент двухосности напряженного состояния, определяемый следующим образом: $\eta=0$ - для растягивающих продольных напряжений, $\eta=$ $1.15\left[\sigma_{\text {кц }}\right] / \mathrm{R}_{1}-$ для сжимающих продольных напряжений.

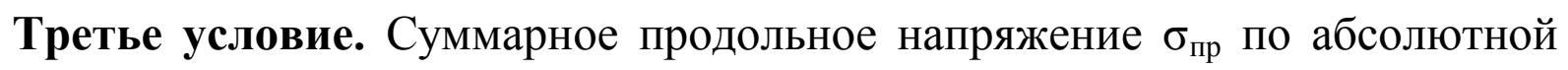
величине не должно превышать допустимого значения $[\sigma]_{3}$, определяемого по формуле:

$$
\sigma_{\text {пр }} \leq\left[\sigma_{\text {пр }}\right]_{3}=\psi_{3} \cdot \mathrm{S} .
$$

Здесь $\mathrm{S}=\sigma_{\mathrm{T}} \cdot \mathrm{m} / 0.9 \mathrm{k}_{\mathrm{H}}$; коэффициенты $\mathrm{m}$ и $\mathrm{k}_{\text {н }}$ определены выше ; $\psi_{3}-$ коэффициент, учитывающий двухосное напряженное состояние стенки трубопровода, определяемый по формуле:

$$
\psi_{3}=\sqrt{ } 1-075 \omega^{2}-0.5 \omega,
$$

где $\omega$ - коэффициент двухосности напряженного состояния, определяемый 
следующим образом : $\omega=0$ - для растягивающих суммарных продольных напряжений $\sigma_{\text {пр }} ; \omega=\sigma_{\text {кц }} / \mathrm{S}$ - для сжимающих продольных напряжений $\sigma_{\text {пр }}$.

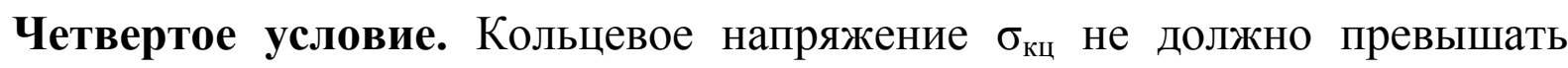
допустимого значения $\sigma_{\text {кц }}$, определяемого по формуле:

$$
\sigma_{\text {кц }} \leq\left[\sigma_{\text {кц }}\right]_{4}=\mathrm{S} .
$$

Значение $\mathrm{S}$ определено выше

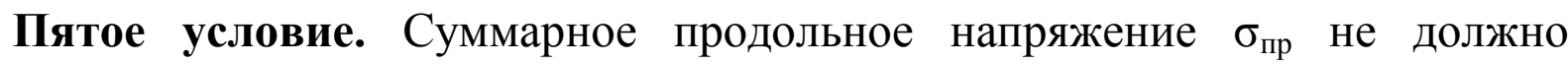
превышать по абсолютному значению 0.7 от предела текучести металла:

$$
\sigma_{\text {пр }} \leq\left[\sigma_{\text {пр }}\right]_{5}=0 . .7 \sigma_{\text {т. }}
$$

Дефектность и старение металла. Если на стенке трубопровода обнаружены дефекты, то по специальным методикам необходимо определить коэффициенты ослабления стенки трубы в кольцевом и продольном направлениях $\sigma_{\text {кц }}$ и $\sigma_{\text {пр }}$.

Старение металла труб при длительной эксплуатации трубопровода учитывается введением коэффициента деформационного старения $\mathrm{K}_{\mathrm{S}}$, определяемого по формуле

$$
\mathrm{K}_{\mathrm{S}}=1+0.025 \mathrm{C}_{\text {экв }} \cdot \mathrm{t},
$$

где $\mathrm{t}$ - время эксплуатации трубопровода (годы); $\mathrm{C}_{\text {экв }}$ - углеродный эквивалент металла, выраженный в процентах.

Предельные значения кольцевых и продольных напряжений должны быть снижены с учетом коэффициентов $\alpha_{\text {кц }}, \alpha_{\text {пр }}, \mathrm{K}_{\mathrm{S}}$ следующим образом:

$$
\begin{gathered}
\sigma_{\text {кц }} \leq \frac{\alpha_{\text {ки }}}{k_{S}}\left[\sigma_{\text {кц }}\right]_{1}=\frac{\alpha_{к u}}{\kappa_{s}} \cdot \frac{R_{1}}{n_{P}} \cdot \psi_{1}, \\
\sigma_{\text {пр }} \leq \frac{\alpha_{n p}}{\kappa_{S}}\left[\sigma_{\text {пр }}\right]_{2}=\frac{\alpha_{n p}}{k_{s}} \cdot \mathrm{R}_{1} \cdot \psi_{2}, \\
\sigma_{\text {пр }} \leq \frac{\alpha_{n p}}{\kappa_{S}}\left[\sigma_{\text {пр }}\right]_{3}=\frac{\alpha_{n p}}{k_{s}} \cdot \psi_{3} \cdot \mathrm{S}, \\
\sigma_{\text {кц }} \leq \frac{\alpha_{\text {кu }}}{k_{S}}\left[\sigma_{\text {кц }}\right]_{4}=\frac{\alpha_{k u}}{k_{S}} \cdot \mathrm{S},
\end{gathered}
$$




$$
\sigma_{\text {пр }} \leq \frac{\alpha_{n p}}{k_{s}}\left[\sigma_{\text {пр }}\right]_{5}=\frac{\alpha_{n p}}{k_{s}} \cdot 0.7 \sigma_{\mathrm{T}} .
$$

Расчет прогнозной инженерной оценки остаточного (рабочего) ресурса с учетом деформационного старения металла производится в следующей последовательности:

a) оценивается состояние трубопровода, для чего выполняют следующие операции:

- диагностируются дефекты и толщина стенки трубопровода;

- измеряются технические изменения НДС трубопровода.

б) создается расчетная модель;

в) расчитывается остаточный ресурс.

Для определения допустимого внутреннего давления используется специальная компьютерная программа. Алгоритм решения состоит из следующих этапов:

1. Ввести исходные данные:

- D - диаметр трубы, м;

- $\delta_{\mathrm{T}^{-}}$толщина стенки трубы, м;

- $\sigma_{\mathrm{T}^{-}}$предел текучести металла, Па;

- $\sigma_{\mathrm{B}}$ - предел прочности металла, Па;

- $\mathrm{E}=206 \cdot 10^{9}$ Па - модуль упругости металла трубы;

- $\mu=03$ - коэффициент Пуассона;

- $\alpha=12 \cdot 10^{-6} 1 /$ град - коэффициент теплового расширения металла;

- $\mathrm{T}_{\mathrm{c}}$ - температура укладки трубопровода, град;

- $\mathrm{T}_{\ni}$ - температура эксплуатации или ремонта, град;

- $\mathrm{m}$ - коэффициент условий работы;

- $\mathrm{k}_{1}$ и $\mathrm{k}_{2}$ - коэффициент надежности по материалу;

- $\mathrm{k}_{\mathrm{H}}$ - коэффициент надежности по назначению трубопровода; 
- $\mathrm{P}_{0}$ и $\Delta \mathrm{P}$ - начальное внутреннее давление и заданная точность определения давления, Па;

- $\mathrm{n}$ - коэффициент надежности по нагрузке - внутреннему рабочему давлению в трубопроводе;

- $\alpha_{\text {кц }}$ и $\alpha_{\text {кц }}$ - коэффициенты ослабления трубы от дефекта в кольцевом и продольном направленияхж

- $\mathrm{t}$ - срок эксплуатации трубопровода.

2. Вычислить расчетные сопротивления металла растяжению (сжатию) $\mathrm{R}_{1}$ и $\mathrm{R}_{2}$ (формулы см. выше).

3. Вычислить кольцевые и продольные напряжения от действия первой группы сил - внутреннего давления и температурного воздействия :

$$
\begin{aligned}
& \sigma_{\text {кц(1) }}=\left[\mathrm{nP}\left(\mathrm{D}-2 \delta_{\mathrm{T}}\right)\right] / 2 \delta_{\mathrm{T}}, \\
& \sigma_{\text {пр(1) }}=\mu \sigma_{\text {кц(1) }}-\mathrm{E} \alpha\left(\mathrm{T}_{\mathrm{c}}-\mathrm{T}_{\ni}\right) .
\end{aligned}
$$

4. Вычислить суммарное кольцевое напряжение $\Sigma \sigma_{\text {кц }}=\sigma_{\text {кц(1) }}$.

5. Вычислить $\sigma_{\text {пр(2) }}$ и $\sigma_{\text {пр(3) }}$ - напряжения от изгиба в вертикальном и горизонтальном направлениях.

6. Вычислить с учетом знаков минимальное и максимальное значения продольных напряжений по формулам:

$$
\begin{aligned}
& \min \sigma_{\text {пр }}=\sigma_{\text {пр }(1)}-\sqrt{\left(\sigma_{\text {пр }(2)}\right)^{2}+\left(\sigma_{\text {пр}(3)}\right)^{2},} \\
& \min \sigma_{\text {пр }}=\sigma_{\text {пр(1) }}+\sqrt{\left(\sigma_{\text {пр}(2)}\right)^{2}+\left(\sigma_{\text {пр}(3)}\right)^{2} .}
\end{aligned}
$$

7. Вычислить вспомогательные величины $\xi$ и $\psi$ по формулам:

$$
\begin{gathered}
\xi=0, \text { если } \sigma_{\text {пр }} \geq 0 ; \\
\xi=-\left(\min _{\text {пр }}\right) / \mathrm{R}_{1}, \text { если } \min \sigma_{\text {пр }}<0 . \\
\psi_{1}=\sqrt{1-0.75 \xi^{2}-0.5 \xi .}
\end{gathered}
$$

8. Вычислить вспомогательные величины $\eta$ и $\psi 2$ по формулам, причем $\eta=0$, если $\sigma_{\text {пр }} \geq 0$ :

$$
\begin{gathered}
\xi=1.15 \sigma_{\text {кц }} / \mathrm{R}_{1}, \text { если } \sigma_{\text {пр }}<0 ; \\
\psi_{2}=\sqrt{1-0.75 \eta^{2}-0.5 \eta .}
\end{gathered}
$$


9. Вычислить вспомогательные величины $\mathrm{S}, \omega$ и $\psi_{3}$ по формулам:

$$
\begin{gathered}
\mathrm{S}=\left(\sigma_{\mathrm{T}} \cdot \mathrm{m}\right) / 0.9 \mathrm{k}_{\mathrm{H}} ; \\
\omega=0, \text { если } \sigma_{\text {пр }} \geq 0 ; \\
\omega=\sigma_{\text {кц }} / \mathrm{S}, \text { если } \sigma_{\text {пр }}<0 ; \\
\psi_{3}=\sqrt{ } 1-0.75 \omega^{2}-0.5 \omega .
\end{gathered}
$$

10. Определить коэффициенты ослабления от дефектов и коэффициент старения металла $\mathrm{k}_{\mathrm{c}}$.

11. Проверить условия прочности и устойчивости (пять условий приведены выше).

12. Если все условия прочности и устойчивости удовлетворяются, то следует увеличить пробное давление на $\Delta \mathrm{P}$ и повторить все расчеты 3 - 10 .

13. Если хотя бы одно условие не удовлетворяется, то за решение $\mathrm{P}$ принять значение давления на предыдущем цикле алгоритма.

Для расчета ресурса трубопроводов используем результаты многолетних наблюдений и технической диагностики нефтепромысловых коммуникаций (свыше 12 тыс. измерений) на Самотлорском месторождении (Западная Сибирь, Россия). Как показали данные многолетнего мониторинга, особенно подвержены коррозионно-механическим повреждениям выкидные линии от нефтяных скважин до автоматизированных групповых замерных установок, несмотря на использование ингибиторов коррозии .

В таблице в качестве примера приведены результаты расчетов допустимого внутреннего давления участков трех обследованных трубопроводов с учетом их характеристик. Расчеты выполнены при коэффициентах ослабления и старения $\alpha_{\text {пр }} / \mathrm{K}_{\mathrm{S}}$, соответствующих сроку эксплутации трубопроводов и их дефектности.

Расчеты показывают, что наименьшей прочностью обладает старый трубопровод, эксплуатируемый на объектах НГДУ "Надвирнанафта" ВАТ “Укрнафта", для которого допустимое внутреннее давление равно 4.87 МПа.

При проведении оценки применимости обследованных трубопроводов с 
целью продления их срока эксплуатации с давлением среды 4 МПа, сравнения проводили с требованиями СНиП 2.05.06-85 “Промысловые трубопроводы” и других нормативных и научно-технических документов.

Результаты расчетов допустимого внутреннего рабочего давления при эксплуатации трубопроводов ВАТ "Укрнафта"

\begin{tabular}{|c|c|c|c|c|c|c|}
\hline $\begin{array}{l}\text { Марка } \\
\text { стали }\end{array}$ & $\begin{array}{c}\text { Диаметр, } \\
\text { мм }\end{array}$ & $\begin{array}{l}\text { Толщина } \\
\text { стенки м }\end{array}$ & $\begin{array}{c}\text { Предел } \\
\text { текучести, } \\
\text { МПа }\end{array}$ & $\begin{array}{c}\text { Предел } \\
\text { прочности, } \\
\text { МПа }\end{array}$ & $\begin{array}{c}\text { Коэффици } \\
\text { ент } \\
\text { ослаблени } \\
\text { я от } \\
\text { старения }\end{array}$ & $\begin{array}{c}\text { Допустим } \\
\text { ое } \\
\text { внутренне } \\
\text { е } \\
\text { давление, } \\
\text { МПа }\end{array}$ \\
\hline $17 Г С$ & 530 & 7.2 & 372.4 & 509.6 & 0.787 & 4.97 \\
\hline Ст10 & 426 & 8.3 & 205.8 & 392 & 0.787 & 5.48 \\
\hline Ст20 & 426 & 8.3 & 345 & 455.7 & 0.787 & 6.46 \\
\hline 17Г1СУ & 508 & 9.8 & 310.7 & 519.4 & 0.781 & 7.28 \\
\hline 14ХГС & 529 & 9 & 338.1 & 509.6 & 0.781 & 6.22 \\
\hline $10 \Gamma 2 \mathrm{C} 1$ & 529 & 9 & 372.4 & 519.4 & 0.781 & 6.34 \\
\hline $09 Г 2 \mathrm{C}$ & 529 & 12 & 294 & 346.9 & 0.781 & 6.54 \\
\hline \multicolumn{7}{|c|}{ Старый нефтепровод ( > 25 лет эксплуатации ) } \\
\hline Ст10 & 508 & 8.1 & 310.6 & 519.4 & 0.641 & 4.87 \\
\hline
\end{tabular}

\section{Выводы}

1. Предложена методика расчета показателей надежности трубопроводных конструкций и инженерных нефтепромысловых коммуникаций и оборудования, которая может использоваться для оценки целесообразности проведения как капитальных ремонтов, так и для разработки конструкторско-технологических решений с целью повышения показателей надежности металлоконструкций нефтяного назначения. 
2. Разработан алгоритм расчета остаточного ресурса промысловых нефтепроводов с учетом трещиностойкости металла, в основу которого положены современные критерии механики разрушения и программы математического моделирования коррозионно-усталостных и циклических разрушений трубных сталей, работающих под давлением и длительное время контактирующих с коррозионно-активными средами в условиях больших перепадов температур $\left(+40 \ldots-60{ }^{0} \mathrm{C}\right)$. 


\section{Литература:}

1. ДСТУ 2860-94. Надійність техніки. Терміни та визначення.

2. Степанов М.Н. Статистические методы обработки результатов механических испытаний. Справочник: - М.:Машиностроение.- 1985.

3. ДСТУ 3004-95 Надійність техніки Методи оцінки показників надійності за експериментальними даними

4. Львовский Е.Н. Статистические методы построения эмпирических формулул. - М.: Высшая школа.- 1988.

5. Макаренко В.Д. Надежность нефтегазопромысловых систем. -Челябинск: изд-во ЦНТИ. -2006. - 826 с.

6. Проников А.С. Надежность машин. - М.:Машиностроение, 1978. - 592 с.

7. Бородавкин П.П. Подземные трубопроводы / Проектирование и строительство.- М.: Недра , 1992. - 384 с.

8. Лютак В.П., Бойчук І.Я. Експлуатаційна надійність нафтопроводів в умовах НГДУ “Надвірнанафтогаз” // Нафтова і газова промисловість. 2002. - №2. - C.38-40.

9. Гоник А.А. Коррозия нефтепромыслового оборудования и меры еe предупреждения. -М.: Недра, 1976. - 235 с.

10. Гумеров А.Г., Ямалеев К.М., Журавлев Г.В. и др. Трещиностойкость металла труб нефтепроводов. - М.: ООО “Недра-Бизнесцентр”, 2001. - 231 c.

11. Усталостная и циклическая трещиностойкость конструкционных материалов / О.Н.Романив, С.Я.Ярема, Г.Н.Никифорчин и др.// Механика разрушения и прочность материалов. Справ. пособие в 4-х т. Под ред. В.В.Панасюка. Т.4. - Киев: Наукова думка, 1990. 